\title{
Morphologic and molecular study of lung cancers associated with idiopathic pulmonary fibrosis and other pulmonary fibroses
}

Alice Guyard', Claire Danel', Nathalie Théou-Anton², Marie-Pierre Debray ${ }^{3}$, Laure Gibault ${ }^{4}$, Pierre Mordant ${ }^{5}$, Yves Castier ${ }^{5}$, Bruno Crestani ${ }^{6,7}$, Gérard Zalcman ${ }^{8,9}$, Hélène Blons ${ }^{10}$ and Aurélie Cazes ${ }^{1,7^{*}}$ (D)

\begin{abstract}
Background: Primitive lung cancers developed on lung fibroses are both diagnostic and therapeutic challenges. Their incidence may increase with new more efficient lung fibrosis treatments. Our aim was to describe a cohort of lung cancers associated with idiopathic pulmonary fibrosis (IPF) and other lung fibrotic disorders (non-IPF), and to characterize their molecular alterations using immunohistochemistry and next-generation sequencing (NGS).

Methods: Thirty-one cancer samples were collected from 2001 to 2016 in two French reference centers for pulmonary fibrosis - 18 for IPF group and 13 for non-IPF group. NGS was performed using an ampliseq panel to analyze hotspots and targeted regions in 22 cancer-associated genes. ALK, ROS1 and PD-L1 expressions were assessed by immunohistochemistry.
\end{abstract}

Results: Squamous cell carcinoma was the most frequent histologic subtype in the IPF group (44\%), adenocarcinoma was the most frequent subtype in the non-IPF group (62\%). Forty-one mutations in 13 genes and one EGFR amplification were identified in 25 samples. Two samples had no mutation in the selected panel. Mutations were identified in TP53 $(n=20)$, MET $(n=4), \operatorname{BRAF}(n=3)$, FGFR3, PIK3CA, PTEN, STK11 $(n=2)$, SMAD4, CTNNB1, DDR2, ERBB4, FBXW7 and KRAS $(n=1)$ genes. No ALK and ROS1 expressions were identified. PD-L1 was expressed in 10 cases (62\%) with only one (6\%) case >50\%.

Conclusions: This extensive characterization of lung fibrosis-associated cancers evidenced molecular alterations which could represent either potential therapeutic targets either clues to the pathophysiology of these particular tumors. These findings support the relevance of large molecular characterization of every lung fibrosis-associated cancer.

Keywords: Idiopathic pulmonary fibrosis, Fibrosis-associated lung cancer, Next-generation sequencing

\section{Background}

Idiopathic pulmonary fibrosis (IPF) is a chronic parenchymal lung disease of severe prognosis, with a median survival of about 3 years from diagnosis [1]. An increased incidence of lung cancer has been described in IPF patients, with a significantly adverse impact on survival [2-6]. IPF and lung cancer are both strongly associated with tobacco-smoking. Incidence of lung

\footnotetext{
*Correspondence: aurelie.cazes@aphp.fr

'Département de Pathologie, Hôpital Bichat-Claude Bernard, Assistance

Publique-Hôpitaux de Paris, 46 rue Henri Huchard, 75018 Paris, France

${ }^{7}$ INSERM U1152, DHU FIRE, Labex Inflamex, Université Paris- Diderot, Paris,

France

Full list of author information is available at the end of the article
}

cancer is also increased in non-idiopathic pulmonary fibrosis suggesting a role for inflammation and fibrosis in the development of lung tumors [7]. Common pathogenic pathways and epigenetic alterations have been described in both IPF and cancer but specific molecular analysis of lung fibrosis-associated tumors has not been published so far [8].

Lung cancer in IPF patients is a therapeutic challenge as both surgery and radiotherapy are limited by lung dysfunction and are at high risk of respiratory exacerbation. Moreover chemotherapy can also be deleterious [5, 9]. However, over the past decade a better knowledge of lung cancer biology led to major changes in the management 
of lung cancer patients. Targeted therapies based on biomarkers have shown clinical success. Genetic alterations differ according to histologic subtypes. In adenocarcinoma (ADC), the most common cancer type, molecular characterization is now an established procedure before any therapeutic decision [10]. In squamous cell carcinoma (SCC), some targets have been identified but need to be validated [11]. Molecular alterations in oncogenes may confer constitutive activation and oncogenic addiction as for EGFR, the first target identified in lung ADC. More recently mutated BRAF and MET were also demonstrated to be addictive oncogenes. Finally, gene fusions, for instance $A L K$ and ROS1 are other molecular mechanisms leading to oncogene activation and are validated targets [12]. In parallel identification of the tumor immune-evasion mechanisms is the basis for innovative therapies, particularly targeting the PD-1/ PD-L1 pathway. Although in need of standardization, PD-L1 expression as detected by immunohistochemistry may be a predictive biomarker of anti PD-1/PDL1 drug's efficacy [13].

The aim of this study was to describe a retrospective cohort of lung cancers developed on IPF and other pulmonary fibroses, and to search for molecular alterations that could either represent therapeutic targets or specific oncogenic pathways in these interstitial lung diseases (ILD).

\section{Methods}

\section{Patients and tumors}

Cases of lung fibrosis-associated lung cancer diagnosed between 2001 and 2016 were identified from clinical and pathological databases of Bichat-Claude Bernard and Georges Pompidou University hospitals (Paris, France), which are both "Competence Centers for rare pulmonary disorders". Formalin-fixed and paraffin-embedded (FFPE) samples were retrieved from Pathology department archives. Two pathologists (AC, AG) reviewed all samples to confirm diagnoses of lung fibrosis and cancer. Cancers were classified according to the 2015 WHO Classification of Lung Tumors [14]. IPF and Idiopathic Interstitial Pneumonias were diagnosed according to American Thoracic Society-European Respiratory Society consensus criteria $[1,15]$. The relationship between tumor and UIP lesions was assessed on 2 slides/tumor on surgical cases of the IPF group. This study was reviewed and approved by the CEERB Paris Nord ethics committee, under the number 16-007.

\section{Next-generation sequencing}

The percentage of tumor cells was assessed by two pathologists (AC, AG), in a macrodissection area if required. DNA extraction from FFPE tissues was performed using Maxwell 16 (Promega, Fitchburg, Wisconsin). DNA was quantified by Qubit ${ }^{\circ} 2.0$ Fluorometer (Qubit ${ }^{\circ}$ dsDNA BR Assay kit-Life Technologies-Thermo Fisher Scientific, Saint Aubin, France). Sequencing libraries were prepared from tumor FFPE DNA using Ion AmpliSeq ${ }^{\text {tu }}$ Colon and Lung Cancer Research Panel V2 (Life Technologies-Thermo Fisher Scientific). This panel targets over 500 hotspot mutations in 22 colon and lung cancer-associated genes: $A K T$ BRAF CTNNB1 EGFR ERBB2 ERBB4 FBXW7 FGFR1 FGFR2 FGFR3 KRAS MET NOTCH1 NRAS PIK3CA PTEN SMAD4 STK11 TP53 ALK DDR2 MAP2K1. The multiplex barcoded libraries were generated with Ion AmpliSeq Library kit from 3- $\mu \mathrm{L}$ of DNA corresponding to 10-30ng. Using NGS data, we developed an algorithm that was used to test the presence of gene amplifications in this series. Amplifications were subsequently validated by qPCR.

MET mutations in the intronic region before the exon 14 were researched in 3 samples (P15, P24, P30) by HRM PCR (LC480, Roche, Basel, Switzerland) followed by Sanger sequencing (abi3130, Thermo Fisher Scientific, Waltham, Massachusetts, USA), using two amplicons of 200 and $212 \mathrm{bp}$ around splice sites (at least 10 bp upstream and downstream).

Mutations were referred to the COSMIC database [16]. Pathogenicity prediction was studied using SIFT, Mutation Taster, PolyPhen and UMD pathogenicity prediction softwares [17-20].

\section{Immunohistochemistry}

Immunohistochemistry was performed on fresh $5-\mu \mathrm{m}$ sections from FFPE blocks on Leica BOND-MAX (Leica Biosystems, Buffalo Grove, IL) automated staining system. Briefly, slides were deparaffinized and subjected to antigen retrieval in a $\mathrm{pH}=9$ buffer. Primary antibodies (ALK - clone 5A4 - Abcam, Cambridge, UK, 1:50 dilution; ROS-1 - clone D4D6 - Genemed Biotechnologies, San Francisco, CA, 1:100 dilution; PD-L1 - clone E1L3N Cell Signaling Technology, Danvers, MA, 1:400 dilution) were incubated for 60,60 and 20 min respectively. Revelation was performed with Leica BOND-MAX detection kits. ALK and ROS1 results were interpreted as positive or negative. PD-L1 result was expressed as the percentage of stained tumor cells.

\section{Statistical analysis}

Continuous variables are described by their mean and $\mathrm{SD}$, and compared by use of Student's $t$-test. Categorical variables are described by percentages and compared by Fisher's exact test. Statistical analysis used Prism 5 (GraphPad Software, La Jolla, CA). P 0.05 was considered statistically significant. 


\section{Results}

\section{Patients}

Thirty-one tumor samples were collected from 30 patients (Table 1). Eighteen were collected from patients diagnosed with IPF and 13 from patients suffering from other lung fibrotic disorders: connective tissue disease-associated interstitial lung disease (CTD-ILD) $n=6$, idiopathic non-specific interstitial pneumonia $n=2$, pneumoconiosis $n=4$, drug-induced lung fibrosis $n=1$.

Men predominate in both groups $(89 \%$ in IPF group and $77 \%$ in non-IPF group, $n=0.62$ ). No difference was

Table 1 Clinical features

\begin{tabular}{|c|c|c|c|c|c|c|c|c|}
\hline Patient & Gender & Age (years) & Tobacco (P-Y) & Disease & CT-scan & Cancer type & Cancer location & Sampling site and mode \\
\hline \multicolumn{9}{|c|}{ Idiopathic pulmonary fibrosis } \\
\hline P1 & M & 86 & $<5$ & IPF & UIP & SCC & peripheral & Lung, biopsy \\
\hline P2 & $\mathrm{F}$ & 63 & 40 & IPF & UIP & SCC & peripheral & Lung, biopsy \\
\hline P3 & M & 60 & NP & IPF & UIP & SCC & peripheral & Lung, surg. resec. \\
\hline P4 & M & 55 & 40 & IPF & UIP & SCC & peripheral & Lung, surg. resec. \\
\hline P5 & M & 41 & 30 & IPF & UIP & SCC & peripheral & Lung, biopsy \\
\hline P6 & M & 69 & 45 & $\mathrm{IPF}$ & UIP & SCC & proximal & LN, EBUS \\
\hline P7 & M & 75 & 30 & IPF & UIP & SCC & peripheral & Lung, surg. resec. \\
\hline P8 & M & 66 & yes (NS) & likely IPF & UIP & SCC & peripheral & Lung, surg. resec. \\
\hline P9 & M & 68 & 20 & IPF & UIP & $A D C$ & peripheral & Lung, biopsy \\
\hline P10 & $\mathrm{F}$ & 56 & 35 & IPF & UIP & $A D C$ & peripheral & Lung, biopsy \\
\hline P3 & M & 61 & NS & IPF & UIP & $A D C$ & peripheral & Lung, autopsy \\
\hline P11 & M & 62 & 0 & IPF & UIP & $A D C$ & peripheral & Pleural liquid \\
\hline P12 & M & 58 & 50 & IPF & UIP & $A D C$ & peripheral & Lung, surg. resec. \\
\hline P13 & M & 64 & 40 & likely IPF & UIP & $A D C$ & peripheral & Lung, surg. resec. \\
\hline P14 & M & 73 & 55 & IPF & UIP & ADS & proximal & Lung, surg. resec. \\
\hline P15 & M & 67 & 10 & IPF & UIP & ADS & peripheral & Lung, surg. resec. \\
\hline P16 & M & 57 & 60 & likely IPF & UIP & LCNEC & peripheral & LN, biopsy \\
\hline P30 & M & 51 & 30 & IPF & UIP & $\mathrm{SmCC}$ & peripheral & Lung, biopsy \\
\hline \multicolumn{9}{|c|}{ Connective Tissue Disease-Interstitial Lung Disease } \\
\hline P18 & M & 57 & 40 & RA & NSIP & SCC & proximal & Lung, surg. resec. \\
\hline P20 & $\mathrm{F}$ & 55 & 10 & RA & UIP & $A D C$ & peripheral & Lung, surg. resec. \\
\hline P21 & M & 69 & 100 & RA & UIP & $A D C$ & peripheral & Lung, surg. resec. \\
\hline P24 & M & 62 & 40 & RA & NSIP & ADS & peripheral & Lung, surg. resec. \\
\hline P23 & M & 66 & 30 & antisynthetase sd & NSIP & $A D C$ & peripheral & LN, biopsy \\
\hline P22 & $\mathrm{F}$ & 59 & 0 & scleroderma & UIP & $A D C$ & peripheral & Lung, surg. resec. \\
\hline \multicolumn{9}{|c|}{ Non-specific interstitial pneumonia } \\
\hline P25 & M & 69 & 70 & NSIP & NSIP & $A D C$ & peripheral & Lung, surg. resec. \\
\hline P26 & $\mathrm{F}$ & 54 & 60 & NSIP & NSIP & $A D C$ & peripheral & Lung, surg. resec. \\
\hline \multicolumn{9}{|c|}{ Pneumoconiosis } \\
\hline P17 & M & 64 & 50 & pneumoconiosis & Em-UIP & SCC & peripheral & Lung, surg. resec. \\
\hline P27 & M & 59 & 17 & asbestosis & UIP & $A D C$ & peripheral & Lung, biopsy \\
\hline P19 & M & 58 & yes (NS) & likely asbestosis & UIP & SCC & peripheral & Lung, biopsy \\
\hline P29 & M & 73 & 50 & asbestosis & Em-UIP & SmCC & peripheral & Lung, biopsy \\
\hline \multicolumn{9}{|c|}{ Drug-induced lung fibrosis } \\
\hline P28 & M & 87 & 60 & NC (amiodarone?) & ILD & $A D C$ & peripheral & Lung, biopsy \\
\hline
\end{tabular}

$A D C$ adenocarcinoma, $A D S$ adenosquamous carcinoma, EBUS endobronchial ultrasound, Em emphysema, IPF idiopathic pulmonary fibrosis, $L C N E C$ large cell neuro-endocrine carcinoma, LN lymph node, NS not specified, NSIP non-specific interstitial pneumonia, $P-Y$ pack-years, RA rheumatoid arthritis, SCC squamous cell carcinoma, SmCC small cell carcinoma, surg. resec surgical resection, UIP usual interstitial pneumonia 
observed in age $(63+/-9.9$ vs $64+/-9.1, p=0.75)$ and tobacco use (never smoker: $5.5 \%$ vs $7.6 \%, p=0.74$ ).

Samples were collected from surgical resection $(\mathrm{n}=16)$, lung core biopsy $(n=10)$, lymph node core biopsy/cytology $(n=3)$, autopsy $(n=1)$ and pleural fluid $(n=1)$. Age of FFPE material ranged from 0 to 13 years $($ mean $=3.5+/-3.3)$.

\section{Pathologic characterization}

Pathologic characterization is summarized in Table 2. In the IPF group, histologic subtypes were SCC $(n=8,44 \%)$, ADC $(n=6,33 \%)$, adenosquamous carcinoma (ADS) $(n=2$, $11 \%)$, small cell carcinoma (SmCC) $(n=1,6 \%)$ and large cell neuro-endocrine carcinoma (LCNEC) $(n=1,6 \%)$. In the non-IPF group, histologic subtypes were $\operatorname{ADC}(n=8,62 \%)$, $\operatorname{SCC}(n=3,23 \%)$, ADS $(n=1,8 \%)$ and $\operatorname{SmCC}(n=1,8 \%)$.

Six of the 11 SCC (55\%) were keratinizing and one was basaloid (Fig. 1a). In ADC, acinar $(n=6,43 \%)$ and solid ( $n=4,29 \%)$ were the most frequent subtypes, both observed in IPF and non-IPF groups. Papillary $(n=2$, $14 \%)$ subtype was observed in the non-IPF group and mucinous $(n=1,7 \%)$ subtype in the IPF group (Fig. $1 \mathrm{~b})$. A high proportion of tumors were peripheral in both groups: $16 / 18$ (89\%) in IPF group and $12 / 13(92 \%)$ in non-IPF group. In the IPF group, 7/9 surgically removed tumors were developed in close contact with peripheral honeycomb regions (Fig. 1c). Two out of 9 were in contact with emphysema lesions.

\section{Immunohistochemistry}

PD-L1 expression was assessed in all surgical resections and in the autopsy specimen, corresponding to 16 cases (6 SCC, 7 ADC and 3 ADS). Among them, 6 had less than $1 \%$ of stained tumor cells, 3 had $1 \%$ to $<5 \%, 6$ had $5 \%$ to $<50 \%$ and one ADC had more than $50 \%$ of stained tumor cells. Overall, 10 tumors (62\%) should be considered as expressing tumor cell membrane PD-L1 antigen in more than $1 \%$ of cells (Table 2 and Fig. 1d), and one (6\%) with a high level of expression.

ALK and ROS1 expression was assessed in all ADC from surgical resections and autopsy specimen $(n=10)$. For two other patients, ALK expression was assessed during the patient management (P9 and P12). In all tested cases, ALK and ROS1 were negative.

\section{Next-generation sequencing}

In $27 / 31$ samples (87\%), DNA quality was sufficient for proper analysis. The mean coverage was 10,646 (median 5,687 , range from 247.8 to 34,874 ).

NGS results are presented in Tables 3 and 4. One or more mutations were found in 25/27 samples (93\%). Eleven samples (41\%) had one mutation, eight (30\%) two mutations, five (19\%) three mutations, and one (4\%) presented an EGFR gene amplification.
Forty-four molecular alterations were identified in 14 genes. Twenty TP53 mutations were detected (Table 3). Nine molecular alterations were found in four genes coding for tyrosine kinase receptors: point mutations in $M E T$ (4) (Fig. 2a), FGFR3 (2), ERBB4 (1) and DDR2 (1) and one EGFR amplification. Seven mutations were described in the PI3K pathway, involving PIK3CA (3), PTEN (2) and STK11 (2) genes. Four mutations involving the MAPK pathway were identified in BRAF (3) (Fig. 2b) and KRAS (1) (Table 4). Single TP53 mutations were observed in 11 patients. Single mutation in another oncogenic gene was found in one case (MET gene for P22). Multiple oncogenic activations were found in 12 patients.

Mutations classified by histologic subtype are in SCC: TP53 ( $n=8,80 \%), \operatorname{MET}(n=2,20 \%), B R A F, P T E N$, SMAD4, STK11 and FBXW7 $(n=1,10 \%)$; in ADC: TP53 ( $n=6,50 \%), B R A F$ and PIK3CA $(\mathrm{n}=2,17 \%)$, MET, FGFR3, STK11, CTNNB1, ERBB4, KRAS and EGFR amplification $(n=1,8 \%)$. Two mutations of TP53 and one mutation of PIK3CA, MET and DDR2 were found in the 2 ADS.

Mutations analysed according to parenchymal disease subtype are, in IPF group: TP53 $(n=11,73 \%)$, MET $(n=3,20 \%)$, PTEN, SMAD4, FBXW7, STK11, PIK3CA and EGFR amplification ( $n=1,7 \%)$; in nonIPF group: TP53 $(n=8,67 \%), B R A F(n=3,25 \%)$, FGFR3 and PIK3CA $(n=2,17 \%)$, STK11, DDR2, $M E T, K R A S, E R B B 4$ and CTNNB1 $(n=1,8 \%)$.

\section{Discussion}

The aim of this study was to describe a cohort of lung cancers developed on IPF and other pulmonary fibroses, and to characterize their molecular alterations. SCC was the most frequent histologic subtype in our IPF group, as mostly reported in previous studies encompassing a large period of time [3, 21]. This squamous histology could suggest specific oncogenic events in the IPF micro-environment where peripheral honeycombassociated squamous metaplasia and dysplasia has been reported [22]. In contrast, ADC was the most frequent subtype in the heterogeneous non-IPF group, like in the general population. Acinar subtype was the most frequent ADC subtype in our cohort (43\%), and invasive mucinous subtype was rare (7\%), as reported in a 89 idiopathic interstitial pneumonia-associated ADC cases recent Japanese series (35.95\% and $11.24 \%$ respectively), described by Kojima [23]. In another recent Japanese series on 44 UIP-associated ADC reported by Masai, invasive mucinous subtype was predominant $(29.5 \%$ of ADC) [6].

Among the genes assessed in the NGS panel, we detected 43 mutations in 13 genes and an EGFR gene amplification in 25 samples. 
Table 2 Pathological features

\begin{tabular}{|c|c|c|c|c|c|c|c|c|}
\hline \multirow[t]{2}{*}{ Patient } & \multirow[t]{2}{*}{ Cancer type } & \multirow{2}{*}{$\begin{array}{l}\text { Cancer } \\
\text { differenciation }\end{array}$} & \multicolumn{3}{|c|}{ Diagnostic immunohistochemistry (IHC) } & \multicolumn{3}{|c|}{ Therapeutic IHC } \\
\hline & & & TTF1 & $\mathrm{p} 40 / \mathrm{p} 63$ & others & ALK & ROS1 & PDL1 \\
\hline \multicolumn{9}{|c|}{ Idiopathic pulmonary fibrosis } \\
\hline P1 & SCC & keratinizing & / & / & & / & / & / \\
\hline P2 & SCC & nonkeratinizing & TTF1- & p40+ & & / & / & / \\
\hline P3 & SCC & $\begin{array}{l}\text { basaloid, } \\
\text { keratinizing }\end{array}$ & / & p63+ & CK7- & / & / & $<1 \%$ \\
\hline P4 & SCC & keratinizing & TTF1- & p40+ & & / & / & $5 \%$ \\
\hline P5 & SCC & nonkeratinizing & TTF1- & p63+ & NapsinA- CK5/6+ & / & / & / \\
\hline P6 & SCC & keratinizing & TTF1- & p63+ & & / & / & / \\
\hline P7 & SCC & keratinizing & TTF1- & p40+ & & / & / & $10 \%$ \\
\hline P8 & SCC & nonkeratinizing & TTF1- & p40+ & & / & / & $0 \%$ \\
\hline P9 & $A D C$ & acinar & TTF1+ & & CK7+ & neg & / & / \\
\hline P10 & $A D C$ & acinar & TTF1- & p63- & & I & / & / \\
\hline P3 & $A D C$ & solid & TTF1+ & p63- & & neg & neg & $<1 \%$ \\
\hline P11 & $A D C$ & NS & TTF1- & p63- & NapsinA+ & / & / & / \\
\hline P12 & $A D C$ & mucinous & TTF1- & / & CK7+ CK20+ & neg & / & / \\
\hline P13 & $A D C$ & acinar & TTF1+ & p40- & CK7+ CD56- & neg & neg & $1 \%$ \\
\hline P14 & ADS & acinar & TTF1- & p40+ & CK7+ & neg & neg & $20 \%$ \\
\hline P15 & ADS & papillary & TTF1+ & p40+ & & neg & neg & $15 \%$ \\
\hline P16 & LCNEC & / & TTF1- & / & $\begin{array}{l}\text { chromoA+ CD56+ } \\
\text { synapto + CK5/6- }\end{array}$ & / & / & / \\
\hline P30 & SmCC & / & TTF1+ & / & $\begin{array}{l}\text { chromoA+ CD56+ } \\
\text { synapto+ }\end{array}$ & / & / & / \\
\hline \multicolumn{9}{|c|}{ Connective Tissue Disease-Interstitial Lung Disease } \\
\hline P18 & SCC & keratinizing & TTF1- & p40+ & & / & / & $40 \%$ \\
\hline P20 & $A D C$ & papillary & TTF1+ & / & & neg & neg & $<1 \%$ \\
\hline P21 & ADC & solid & TTF1+ & $\mathrm{p} 40-$ & & neg & neg & $70 \%$ \\
\hline P24 & ADS & solid & TTF1+ & p40+ & & neg & neg & $10 \%$ \\
\hline P23 & $A D C$ & solid & TTF1+ & p63- & NapsinA+ & / & / & / \\
\hline P22 & $A D C$ & acinar & TTF1+ & / & CK7+ & neg & neg & $0 \%$ \\
\hline \multicolumn{9}{|c|}{ Non-specific interstitial pneumonia } \\
\hline P25 & $A D C$ & acinar & TTF1+ & p40+ & & neg & neg & $<1 \%$ \\
\hline P26 & $A D C$ & papillary & TTF1+ & / & & neg & neg & $1 \%$ \\
\hline \multicolumn{9}{|c|}{ Pneumoconiosis } \\
\hline P17 & SCC & keratinizing & TTF1- & p40+ & & / & / & $1 \%$ \\
\hline P27 & $A D C$ & solid & TTF1+ & p40+ & & / & / & / \\
\hline P19 & SCC & nonkeratinizing & TTF1- & p63+ & CK5/6+ & / & / & / \\
\hline P29 & SmCC & / & TTF1- & / & CD56+ & / & / & / \\
\hline
\end{tabular}

Drug-induced lung fibrosis

$\begin{array}{llll}\text { P28 ADC } & \text { acinar } & \text { NTF1+ }\end{array}$

We detected TP53 mutations in 8 SCC (80\% of SCC) and $6 \mathrm{ADC}(50 \%$ of $\mathrm{ADC})$, with the same frequency as reported in the literature [11]. We also detected TP53 mutations in all other cancer subtypes. Allelic ratios suggest a loss of the second TP53 allele, as usually in cancers [24]. Detected mutations occurring in the DNA 


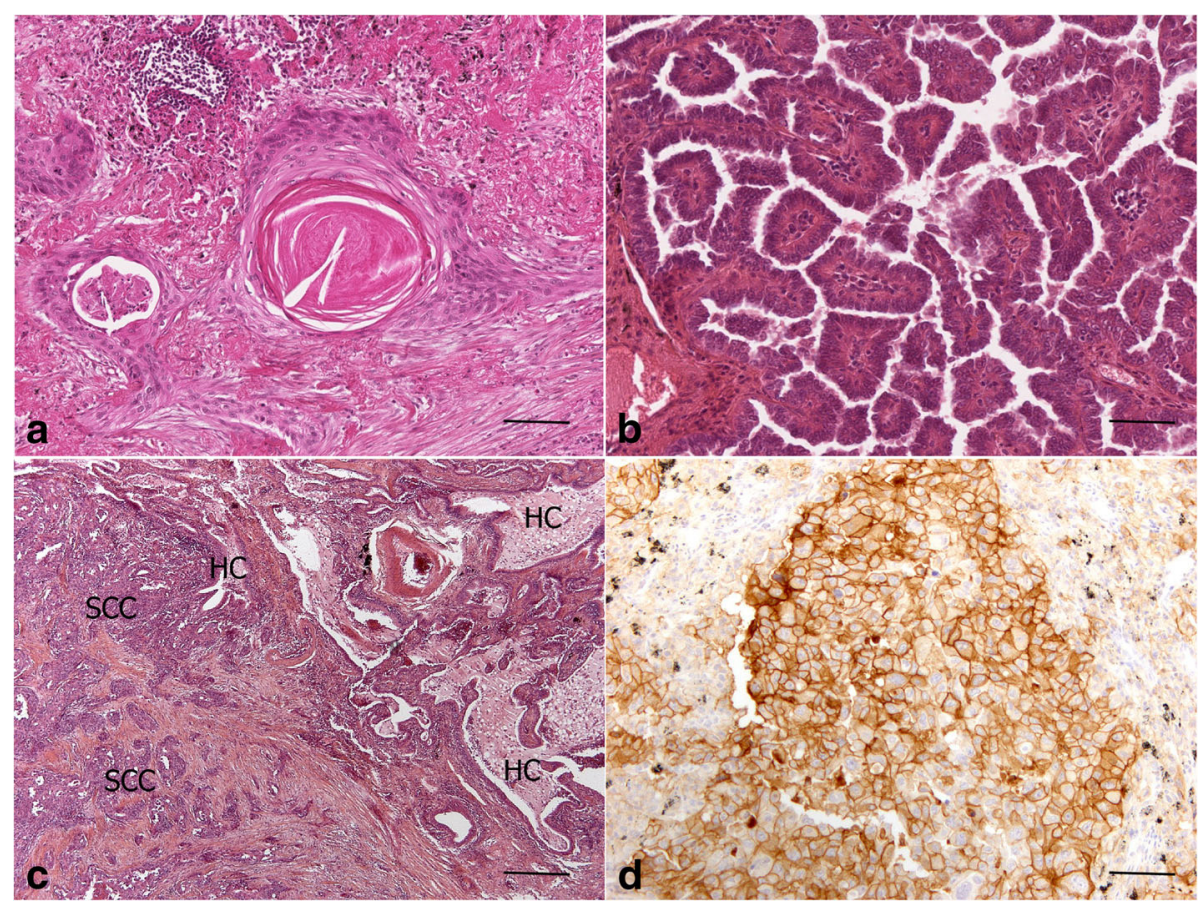

Fig. 1 Pathological and immunohistochemical characteristics of lung tumors. a Keratinising squamous cell carcinoma (P4, HES, x20 objective, scale

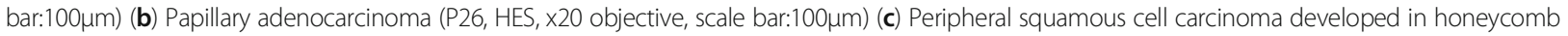

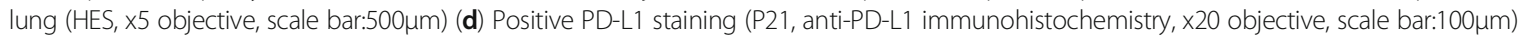

binding domain (from codon 125 to 300), especially the hotspot codons in CpG sites, are similar to those already described, according to the COSMIC public database [16]. More than one third are $\mathrm{G}>\mathrm{T}$ transversions, in accordance with the high proportion of smokers [25]. Thus a specific carcinogenesis process differing from tobacco smoke DNA signature and linked to chronic lung inflammation could not be inferred from this molecular analysis.

Four MET mutations were detected in our cohort: p.Arg359Gln and p.Arg988Cys in SCC (20\%), p.Thr1011Ala in one ADC (8\%) and c.2942-36G > A in one ADS. In the literature, $M E T$ mutations are reported in $2 \%$ to $7 \%$ of lung ADC and in $1 \%$ of lung SCC [12]. Codon 359 is located within the SEMA domain, involved in binding with the MET-specific ligand HGF. Codons 988 and 1011 are located in the exon 14, and c.2942$36 \mathrm{G}>\mathrm{A}$ in the intronic region before the exon 14, required for negative regulation of MET. Mutations involving exon 14 splicing site have been described in lung ADC, they mostly result in exon 14 skipping and ultimately in MET protein stabilization [12, 26]. Case reports have demonstrated responses to MET-inhibitors in ADC patients with METex14 alterations [26]. METex14 mutations were, so far, not reported in lung SCC. These three exonic mutations have been described as rare polymorphisms. However their functional impact remains unclear as discordant results are obtained with pathogenicity prediction softwares. For instance p.Arg988Cys, although described as a germline polymorphism (rs34589476), has been reported in numerous lung cancers, and its pathogenic role remains elusive, in vitro data supporting functional consequences [27, 28]. Interestingly, in our cohort, three MET mutations occurred in IPF and 1 in CTD-ILD with an UIP pattern on CTscan. Whether these variants represent true oncogenic drivers or significant polymorphisms in the fibrotic process, this could suggest a specific pathway in IPF/UIP lung with activation of the HGF/MET axis [29]. The search for MET mutations in non-tumoral IPF lung would be mandatory to test these hypotheses. Of note, we looked for mutations in flanking introns of exon 14 in only three cases. Thus we cannot exclude the possibility of more MET mutations. Whether such alterations could be targetable would deserve specific clinical trials.

A p.Trp259Arg DDR2 mutation was observed in an ADS. In the literature, DDR2 mutations are found in $4 \%$ of lung SCC and in 1\% of lung ADC, without hotspot mutations. Clinical response to dasatinib was reported in rare case-reports of patients with lung SCC [30].

No mutation of EGFR was observed in our cohort, although reported in $10-15 \%$ of lung ADC [12]. This result, in addition to the absence of ALK and ROS1 rearrangement, is consistent with the predominance of male smokers in our cohort. Three recent Japanese studies also described a significantly lower EGFR mutation frequency in ILD/IPF patients $[5,6,23]$. 


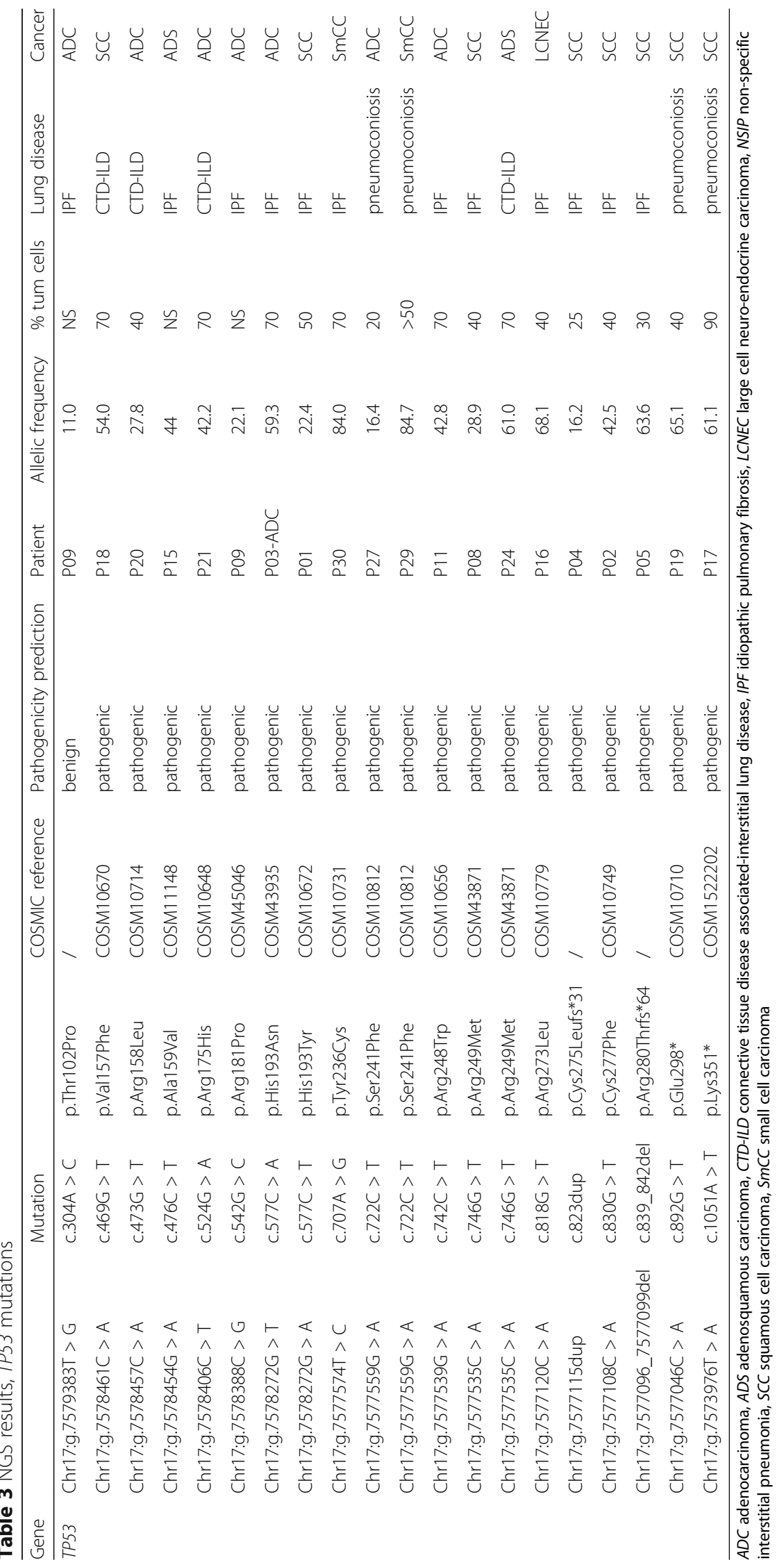




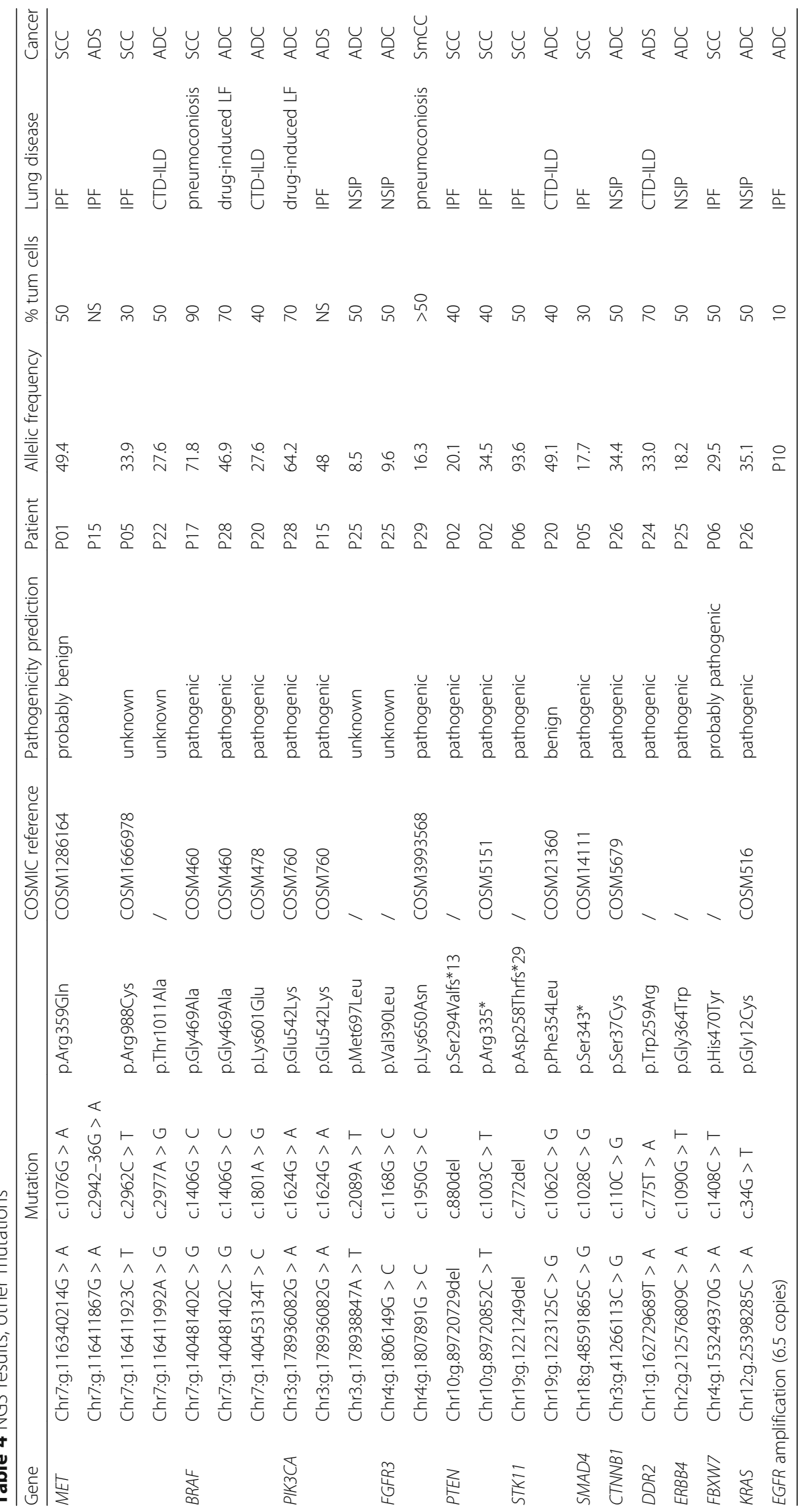




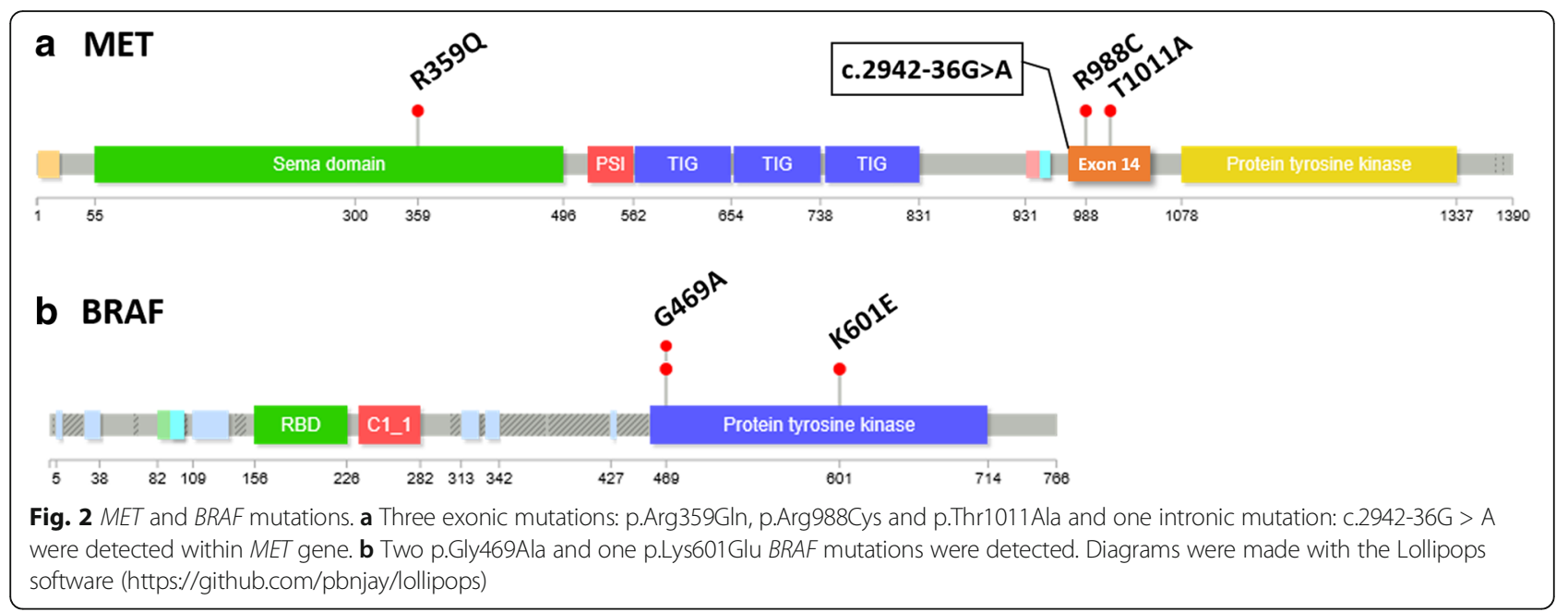

Mutations involving the MAP kinase pathway are frequent in ADC [12]. We described a p.Gly469Ala BRAF mutation in a SCC (10\% of SCC), a p.Lys601Glu and a p.Gly469Ala $B R A F$ mutation in 2 ADC (17\% of ADK). In the literature, $B R A F$ mutations are reported in about $4 \%$ of lung SCC and in $10 \%$ of lung ADC $[11,12]$. BRAF mutations p.Lys601Glu and p.Gly469Ala have already been described in lung ADC. Non-V600E mutations are usual, representing about half of $B R A F$ mutations [31]. Conversely, p.Gly469Ala has never been described in lung SCC. Both are activating BRAF mutations. BRAF and MEK inhibitors can target p.V600E BRAF mutations [31, 32]. Response rates for lung cancer patients with non-V600 mutations are unknown. Only one ADC was KRAS mutated (representing $8 \%$ of adenocarcinomas) whereas KRAS mutations are reported in more than $30 \%$ of lung ADC [12], especially in smokers. While the absence of EGFR mutation could be explained by the high smoking rate in our population, the low incidence of KRAS mutations could suggest the implication of other oncogenic drivers possibly related to the chronic lung injury during the fibrotic process. Interestingly the recent series described by Masai et al. included frequent invasive mucinous ADC (29,5\%), associated with numerous KRAS mutations $(30,2 \%)$ [6]. This could suggest carcinogenesis differences linked to ethnicity or be the reflect of our limited number of patients. However these results were not confirmed by Kojima et al. who reported a low rate of invasive mucinous subtype $(11,24 \%)$ and no difference of KRAS mutation rate between nonUIP-ADC and UIP-ADC [23].

One SMAD4 mutation was found in one SCC-IPF tumors. SMAD4 is a tumor-suppressive gene that can cause cell cycle arrest and apoptosis of epithelial cells, and is inactivated by mutation in over half of pancreatic cancers [33]. It acts as a central mediator in the transforming growth factor- $\beta$ (TGF- $\beta$ ) signalling pathway.
SMAD4 mutations are uncommon in lung cancer, according to COSMIC database. However this signalling pathway, targeted by TGF-beta, could be of particular relevance in a lung fibrosis context. pSer343* predicted as pathogenic is located in the $\mathrm{MH} 2$ region which is implicated in the oligomerization of the protein which is essential for TGFbeta signalling [34].

A p.Ser37Cys CTNNB1 mutation was detected in an ADC (8\%). The codon 37 is a known hotspot mutation, implied in the constitutive activation of the Wnt signalling pathway, and the p.Ser37Cys mutation has been reported in lung ADC [35]. Mutated beta-catenin (CTNNB1) accumulation is followed by translocation to the nucleus and action in a transcriptional complex involving other transcriptional regulators like YAP1 to modulate apoptosis, proliferation or epithelial-mesenchymal transition [36].

A p.His470Tyr FBXW7 mutation was detected in a SCC (10\%). FBXW7 mutations are uncommon in lung cancer, according to COSMIC. FBXW7 is implicated in proteasome degradation of specific substrates and control tumorigenesis, acting on cell cycle, differentiation and apoptosis [37]. It is also involved in epithelial-tomesenchymal transition by controlling mTOR pathway [38]. A p.Arg465His $F B X W 7$ mutation was reported in a lung ADC; the patient benefited from the mTOR inhibitor temsirolimus [39].

Besides molecular targeted therapies, immunotherapy using checkpoint inhibitors is a new efficient therapy against lung cancer. PD-L1 is an immune-checkpoint protein, interacting with its ligand PD-1 expressed by Tcells, used by the tumoral cell to escape the antitumor immune response. Several drugs target the PD-1/PD-L1 interaction. An association between therapeutic response and PD-L1 expression on tumor cells has been described, although it is not a binary predictive marker and the PD-L1 assays need further standardization and validation [13]. PD-L1 expression was assessed in 16 surgical 
cases in the current work. All ADC but one had less than $5 \%$ of stained tumor cells, which, in addition to the pulmonary adverse effects of these molecules, may not plead for a first-line use of immunotherapy in these patients. This has to be investigated in larger series. As far as SCC are concerned PD-L1 expression seems to be less correlated to efficacy, at least in second-line of treatment [40].

\section{Conclusion}

We report here for the first time, to our best knowledge, an extensive pathological and molecular analysis of lung fibrosis-associated lung cancers. We found potentially actionable alterations in MET, FGFR3, ERBB4, DDR2, EGFR, BRAF, PI3KCA genes in various histologic subtypes. While most detected mutations are likely tobacco-associated TP53 mutations, others may suggest alternative oncogenesis mechanisms: notably we found MET, FGFR3, SMAD4 and CTNNB1 mutations, all genes that could potentially be involved in the lung fibrosis process, either participating to epithelial-mesenchymal transition or the regulation or TGF $\beta$ pathway. Conversely, the low prevalence of KRAS mutations, contrasting with the high percentage of smokers, also supports a role for endogenous carcinogenic mechanisms linked to lung fibrosis. Although limited by the size of the cohort, our series shows the feasibility of such systematic molecular characterization, for both therapeutic and pathophysiological purposes. The high mortality of fibrotic lung diseases implies that cancer remains a rare complication since possibly occurring late in the course of fibrosis. Two recently approved drugs, pirfenidone and nintedanib, have been shown to slow IPF progression [41], and are expected to extend survival. If confirmed this may lead to an increase of challenging cancer cases and encourage to perform a large molecular characterization to every lung fibrosis-associated cancer.

\section{Abbreviations}

ADC: Adenocarcinoma; ADS: Adenosquamous carcinoma; CTD-

ILD: Connective tissue disease-associated interstitial lung disease; FFPE: Formalin-fixed and paraffin-embedded; IPF: Idiopathic pulmonary fibrosis; LCNEC: Large cell neuro-endocrine carcinoma; NGS: Next-generation sequencing; SCC: Squamous cell carcinoma; SmCC: Small cell carcinoma

\section{Acknowledgement}

None.

\section{Funding}

Dr Guyard received a research grant from the «Société Française de Pathologie ». No other funding.

\section{Availability of data and materials}

The datasets generated during and/or analysed during the current study are available from the corresponding author on reasonable request.

\section{Authors' contributions}

AG and AC drafted the manuscript, performed histopathological examination of tumors and lung fibroses and molecular and immunohistochemical analyses.
NTA and HB performed the molecular analyses. CD and LG performed the histopathological examination of tumors and lung fibroses. MPD, PM and YC participated in data collection and analyses. GZ, BC, HB and AC participated in the design and coordination of the study and helped to draft the manuscript. All authors have read and approved the final manuscript.

\section{Competing interests}

Pr. Crestani reports grants, personal fees and non-financial support from Boehringer-Ingelheim, Intermune/Roche, Medimmune/Astra Zeneca, personal fees from Sanofi, outside the submitted work. Pr. Zalcman reports personal fees and non-financial support from Roche, Pfizer, personal fees from BMS, Astra-Zeneca, non-financial support from GSK, Lilly, Boehringer-Ingelheim, outside the submitted work. Pr. Blons reports personal fees from Astra-Zeneca, Boehringer, Pfizer, outside the submitted work.

The other authors have no conflict of interest.

Consent for publication

Not applicable.

\section{Ethics approval and consent to participate}

This study was reviewed and approved by the CEERB Paris Nord ethics committee, under the number 16-007. Working retrospectively on archived FFPE tissues we were granted a waiver of consent for dead patients. Alive patients were informed and consent to theranostics work-up of tumoral tissue.

\section{Publisher's Note}

Springer Nature remains neutral with regard to jurisdictional claims in published maps and institutional affiliations.

\section{Author details}

'Département de Pathologie, Hôpital Bichat-Claude Bernard, Assistance Publique-Hôpitaux de Paris, 46 rue Henri Huchard, 75018 Paris, France. ²Département de Génétique, Hôpital Bichat-Claude Bernard, Assistance Publique-Hôpitaux de Paris, 46 rue Henri Huchard, 75018 Paris, France. ${ }^{3}$ Service de Radiologie, Hôpital Bichat-Claude Bernard, Assistance Publique-Hôpitaux de Paris, 46 rue Henri Huchard, 75018 Paris, France. ${ }^{4}$ Service de Pathologie, Hôpital Européen Georges Pompidou, Assistance Publique-Hôpitaux de Paris, 20 rue Leblanc, 75015 Paris, France. ${ }^{5}$ Service de chirurgie vasculaire et thoracique, Hôpital Bichat-Claude Bernard, Assistance Publique-Hôpitaux de Paris, 46 rue Henri Huchard, 75018 Paris, France. ${ }^{6}$ Service de Pneumologie A, Hôpital Bichat-Claude Bernard, Assistance Publique-Hôpitaux de Paris, 46 rue Henri Huchard, 75018 Paris, France. ${ }^{7}$ INSERM U1152, DHU FIRE, Labex Inflamex, Université Paris- Diderot, Paris, France. ${ }^{8}$ Service d'Oncologie Thoracique, CIC1425/CLIP2 Paris-Nord, Université Paris-Diderot, Hôpital Bichat-Claude Bernard, AP-HP, 46 rue Henri Huchard, 75018 Paris, France. ${ }^{9}$ INSERM U830, Institut Curie, Paris, France.

${ }^{10}$ Department of Biochemistry, Pharmacogenetic and Molecular Oncology Unit, Hôpital Européen Georges Pompidou, Assistance Publique - Hôpitaux de Paris, INSERM UMR-S1147, Université Sorbonne Paris Cité, Paris, France.

Received: 27 January 2017 Accepted: 7 June 2017

Published online: 15 June 2017

\section{References}

1. Raghu G, Rochwerg B, Zhang Y, Garcia CAC, Azuma A, Behr J, et al. An Official ATS/ERS/JRS/ALAT Clinical Practice Guideline: Treatment of Idiopathic Pulmonary Fibrosis. An Update of the 2011 Clinical Practice Guideline. Am J Respir Crit Care Med. 2015;192:e3-e19.

2. Le Jeune I, Gribbin J, West J, Smith C, Cullinan P, Hubbard R. The incidence of cancer in patients with idiopathic pulmonary fibrosis and sarcoidosis in the UK. Respir Med. 2007;101:2534-40.

3. Ozawa Y, Suda T, Naito T, Enomoto N, Hashimoto D, Fujisawa T, et al. Cumulative incidence of and predictive factors for lung cancer in IPF. Respirology. 2009;14:723-8.

4. Tomassetti S, Gurioli C, Ryu JH, Decker PA, Ravaglia C, Tantalocco P, et al. The impact of lung cancer on survival of idiopathic pulmonary fibrosis. Chest. 2015;147:157-64.

5. Kanaji N, Tadokoro A, Kita N, Murota M, Ishii T, Takagi T, et al. Impact of idiopathic pulmonary fibrosis on advanced non-small cell lung cancer survival. J Cancer Res Clin Oncol. 2016;142:1855-65. 
6. Masai K, Tsuta K, Motoi N, Shiraishi K, Furuta K, Suzuki S, et al. Clinicopathological, Immunohistochemical, and Genetic Features of Primary Lung Adenocarcinoma Occurring in the Setting of Usual Interstitial Pneumonia Pattern. J Thorac Oncol. 2016;11:2141-9.

7. Daniels CE, Jett JR. Does interstitial lung disease predispose to lung cancer? Curr Opin Pulm Med. 2005;11:431-7.

8. Vancheri C. Common pathways in idiopathic pulmonary fibrosis and cancer. Eur Respir Rev. 2013;22:265-72.

9. Kreuter M, Ehlers-Tenenbaum S, Schaaf M, Oltmanns U, Palmowski K, Hoffmann $\mathrm{H}$, et al. Treatment and outcome of lung cancer in idiopathic interstitial pneumonias. Sarcoidosis Vasc Diffuse Lung Dis. 2015;31:266-74.

10. Barlesi F, Mazieres J, Merlio J-P, Debieuvre D, Mosser J, Lena H, et al. Routine molecular profiling of patients with advanced non-small-cell lung cancer: results of a 1-year nationwide programme of the French Cooperative Thoracic Intergroup (IFCT). Lancet. 2016;387:1415-26.

11. Hammerman PS, Lawrence MS, Voet D, Jing R, Cibulskis K, Sivachenko A, et al. Comprehensive genomic characterization of squamous cell lung cancers. Nature. 2012;489:519-25.

12. Cancer Genome Atlas Research Network. Comprehensive molecular profiling of lung adenocarcinoma. Nature. 2014;511:543-50.

13. Kerr KM, Nicolson MC. Non-small cell lung cancer, PD-L1, and the pathologist. Arch Pathol Lab Med. 2016;140:249-54.

14. Travis WD, Brambilla E, Nicholson AG, Yatabe Y, Austin JHM, Beasley MB, et al. The 2015 world health organization classification of lung tumors: impact of genetic, clinical and radiologic advances since the 2004 classification. J Thorac Oncol. 2015;10(9):1243-60.

15. Travis WD, Costabel U, Hansell DM, King TE, Lynch DA, Nicholson AG, et al. An Official American Thoracic Society/European Respiratory Society Statement: Update of the International Multidisciplinary Classification of the Idiopathic Interstitial Pneumonias. Am J Respir Crit Care Med. 2013;188:733-48.

16. Forbes SA, Beare D, Gunasekaran P, Leung K, Bindal N, Boutselakis H, et al. COSMIC: exploring the world's knowledge of somatic mutations in human cancer. Nucleic Acids Res. 2015;43:D805-11.

17. Vaser R, Adusumalli S, Leng SN, Sikic M, Ng PC. SIFT missense predictions for genomes. Nat Protoc. 2015;11:1-9.

18. Adzhubei I, Jordan DM, Sunyaev SR. Predicting Functional Effect of Human Missense Mutations Using PolyPhen-2. In: Haines JL, Korf BR, Morton CC, Seidman CE, Seidman JG, Smith DR, editors. Curr. Protoc. Hum. Genet. Hoboken: John Wiley \& Sons, Inc; 2013. [cited 2016 Sep 10]. p. 7.20.1-7.20. 41Available from: http://doi.wiley.com/10.1002/0471142905.hg0720s76.

19. Schwarz JM, Rödelsperger C, Schuelke M, Seelow D. MutationTaster evaluates disease-causing potential of sequence alterations. Nat Methods. 2010;7:575-6.

20. Salgado D, Desvignes J-P, Rai G, Blanchard A, Miltgen M, Pinard A, et al. UMDPredictor: A High-Throughput Sequencing Compliant System for Pathogenicity Prediction of any Human cDNA Substitution. Hum Mutat. 2016;37:439-46.

21. Aubry M-C, Myers JL, Douglas WW, Tazelaar HD, Washington Stephens TL, Hartman TE, et al. Primary pulmonary carcinoma in patients with idiopathic pulmonary fibrosis. Mayo Clin Proc. 2002;77:763-70.

22. King TE, Pardo A, Selman M. Idiopathic pulmonary fibrosis. Lancet. 2011;378:1949-61

23. Kojima Y, Okudela K, Matsumura M, Omori T, Baba T, Sekine A, et al. The pathological features of idiopathic interstitial pneumonia-associated pulmonary adenocarcinomas. Histopathology. 2017;70:568-78.

24. Demidenko ZN, Fojo T, Blagosklonny MV. Complementation of two mutant p53: Implications for loss of heterozygosity in cancer. FEBS Lett. 2005;579:2231-5.

25. Hainaut P, Pfeifer GP. Patterns of $\mathrm{p} 53 \mathrm{G}->\mathrm{T}$ transversions in lung cancers reflect the primary mutagenic signature of DNA-damage by tobacco smoke. Carcinogenesis. 2001;22:367-74

26. Frampton GM, Ali SM, Rosenzweig M, Chmielecki J, Lu X, Bauer TM, et al. Activation of MET via Diverse Exon 14 Splicing Alterations Occurs in Multiple Tumor Types and Confers Clinical Sensitivity to MET Inhibitors. Cancer Discov. 2015;5:850-9.

27. Tjin EPM, Groen RWJ, Vogelzang I, Derksen PWB, Klok MD, Meijer HP, et al. Functional analysis of HGF/MET signaling and aberrant HGF-activator expression in diffuse large B-cell lymphoma. Blood. 2006;107:760-8.

28. Ma PC, Kijima T, Maulik G, Fox EA, Sattler M, Griffin JD, et al. c-MET mutational analysis in small cell lung cancer: novel juxtamembrane domain mutations regulating cytoskeletal functions. Cancer Res. 2003;63:6272-81.

29. Crestani B, Marchand-Adam S, Quesnel C, Plantier L, Borensztajn K, Marchal J, et al. Hepatocyte growth factor and lung fibrosis. Proc Am Thorac Soc. 2012;9:158-63.
30. Hammerman PS, Sos ML, Ramos AH, Xu C, Dutt A, Zhou W, et al. Mutations in the DDR2 Kinase Gene Identify a Novel Therapeutic Target in Squamous Cell Lung Cancer. Cancer Discov. 2011;1:78-89.

31. Nguyen-Ngoc T, Bouchaab H, Adjei AA, Peters S. BRAF Alterations as Therapeutic Targets in Non-Small-Cell Lung Cancer. J Thorac Oncol. 2015;10:1396-403.

32. Gautschi O, Milia J, Cabarrou B, Bluthgen M-V, Besse B, Smit EF, et al. Targeted Therapy for Patients with BRAF-Mutant Lung Cancer Results from the European EURAF Cohort. J Thorac Oncol. 2015;10:1451-7.

33. Laklai H, Miroshnikova YA, Pickup MW, Collisson EA, Kim GE, Barrett AS, et al. Genotype tunes pancreatic ductal adenocarcinoma tissue tension to induce matricellular fibrosis and tumor progression. Nat Med. 2016;22:497-505.

34. Miyaki M, Kuroki T. Role of Smad4 (DPC4) inactivation in human cancer. Biochem Biophys Res Commun. 2003;306:799-804.

35. Shigemitsu K, Sekido Y, Usami N, Mori S, Sato M, Horio Y, et al. Genetic alteration of the beta-catenin gene (CTNNB1) in human lung cancer and malignant mesothelioma and identification of a new 3p21. 3 homozygous deletion. Oncogene. 2001;20:4249-57.

36. Baum B, Georgiou M. Dynamics of adherens junctions in epithelial establishment, maintenance, and remodeling. J Cell Biol. 2011;192:907-17.

37. Cao J, Ge M-H, Ling Z-Q. Fbxw7 Tumor Suppressor: A Vital Regulator Contributes to Human Tumorigenesis. Medicine (Baltimore). 2016;95, e2496.

38. Díaz VM, de Herreros AG. F-box proteins: Keeping the epithelial-tomesenchymal transition (EMT) in check. Semin Cancer Biol. 2016;36:71-9.

39. Villaruz LC, Socinski MA. Temsirolimus therapy in a patient with lung adenocarcinoma harboring an FBXW7 mutation. Lung Cancer Amst Neth. 2014;83:300-1.

40. Brahmer J, Reckamp KL, Baas P, Crinò L, Eberhardt WEE, Poddubskaya E, et al. Nivolumab versus Docetaxel in Advanced Squamous-Cell Non-Small-Cell Lung Cancer. N Engl J Med. 2015;373:123-35.

41. Ryerson CJ, Collard HR. Hot off the breath: A big step forward for idiopathic pulmonary fibrosis. Thorax. 2014;69:791-2.

\section{Submit your next manuscript to BioMed Central and we will help you at every step:}

- We accept pre-submission inquiries

- Our selector tool helps you to find the most relevant journal

- We provide round the clock customer support

- Convenient online submission

- Thorough peer review

- Inclusion in PubMed and all major indexing services

- Maximum visibility for your research

Submit your manuscript at www.biomedcentral.com/submit
Biomed Central 